\title{
Performing an Additional Lateral Decubitus PET/CT Scan to Resolve a Respiratory Motion Artifact
}

\author{
Roberto Rinaldi, Luca Camoni, and Domenico Albano \\ Department of Nuclear Medicine, ASST Spedali Civili Brescia, Brescia, Italy
}

\begin{abstract}
Respiratory motion artifacts may affect interpretation of wholebody ${ }^{18}$ F-FDG PET/CT scans, especially when lesions are localized between the liver and the lung. We report a case of a patient with breast cancer who underwent PET/CT after therapy and in whom focal ${ }^{18} \mathrm{~F}-\mathrm{FDG}$ uptake of equivocal interpretation was observed between the liver and the pleura. A subsequent imaging acquisition of the right lateral decubitus showed that the lesion had a pleural location, thus improving the diagnostic accuracy of the PET/CT finding.
\end{abstract}

Key Words: PET/CT; lung; liver; artifact management; respiratory motion

J Nucl Med Technol 2021; 49:84-85

DOI: 10.2967/jnmt.120.251975

\section{B} one, liver, lung, and brain are common metastatic sites in breast cancer and are often evaluated by ${ }^{18} \mathrm{~F}-\mathrm{FDG}$ PET/ CT for a metabolic characterization (1).

\section{CASE REPORT}

A 50-y-old woman with a history of left breast cancer previously treated with mastectomy and axillary lymph node dissection underwent ${ }^{18} \mathrm{~F}$-FDG PET/CT for suspected splenic and lung metastases. PET/CT images were acquired $60 \mathrm{~min}$ after intravenous injection of a $3.5 \mathrm{MBq} / \mathrm{kg}$ dose of ${ }^{18}$ F-FDG on a GE Healthcare Discovery 690 64-slice tomograph (40-mAs low-dose CT, $120 \mathrm{kV}, 2.5 \mathrm{~min}$ per bed position, $256 \times 256$ matrix, $70-\mathrm{cm}$ field of view) and revealed a focal increase in uptake between the liver and the pleura of unclear origin, suggestive of metastatic disease (Fig. 1).

Subsequently, a second segmental acquisition was performed with the patient in the right lateral decubitus position, which allowed displacement of the liver away from the chest wall and showed that the lesion originated from the pleura (Figs. 2A and 2B). A subsequent high-dose CT scan confirmed the pleural location (Fig. 2C).

Received Jun. 23, 2020; revision accepted Sep. 2, 2020.

For correspondence or reprints contact: Roberto Rinaldi, ASST Spedali Civili Brescia, P.le Spedali Civili 1, Brescia 25100, Italy.

E-mail: tsrm.rinaldi@gmail.com

Published online Oct. 5, 2020.

COPYRIGHT (c) 2021 by the Society of Nuclear Medicine and Molecular Imaging.

\section{DISCUSSION}

Respiratory motion on PET/CT scans can cause lesions to be missed or dislocated along the diaphragm, determining a systematic decrease in SUV measurements (2). Also, smalllesion detection can be hampered by diaphragmatic motion, which causes an internal shift in organs that introduces image artifacts, image blurring, and a consequent lower ability to detect (due to the partial-volume effect) and locate lesions. The region between the liver and the lung is particularly sensitive to motion artifacts, with frequent difficulty or errors in attributing findings to specific anatomic territories. Another limitation of PET/CT is the low spatial resolution (about $5 \mathrm{~mm}$ ) of conventional scanners, potentially causing quantitative distortions and reduced sensitivity due to the partial-volume effect (3).

The main technical solutions available to manage respiratory motion artifacts in PET/CT studies are hardware or software technologies provided by several vendors with different definitions (respiratory gating, motion-free, end-expiration, banana artifact management, and datadriven gating), which show a benefit in terms of image quality, quantification, and diagnostic sensitivity. However, patient compliance, long acquisition times, and unavailability of these techniques represent major limitations (4).

In this case, we applied a different acquisition method to manage this artifact. After the whole-body acquisition, the patient was placed in the right lateral decubitus position and a segmental scan was acquired. This positioning permitted separation of the pleura from the liver, showing that the lesion was pleural, and increased diagnostic and quantitative accuracy: the $\mathrm{SUV}_{\max }$ increased from 7 in whole-body scanning to 7.5 in the modified acquisition posture (Figs. $2 \mathrm{~A}$ and $2 \mathrm{~B}$ ). Evaluating the $\mathrm{SUV}_{\text {max }}$, we found the delayed time between the 2 acquisitions to be $6 \mathrm{~min}$; this is a factor to take into account.

\section{CONCLUSION}

This case illustrates a possible way to manage PET/CT scan artifacts during free breathing and the consequent difficult and unclear definition of the anatomic pertinence of focal uptake in peripheral lung regions or, alternatively, in pleura or liver. This approach is simple, cost-free, and 


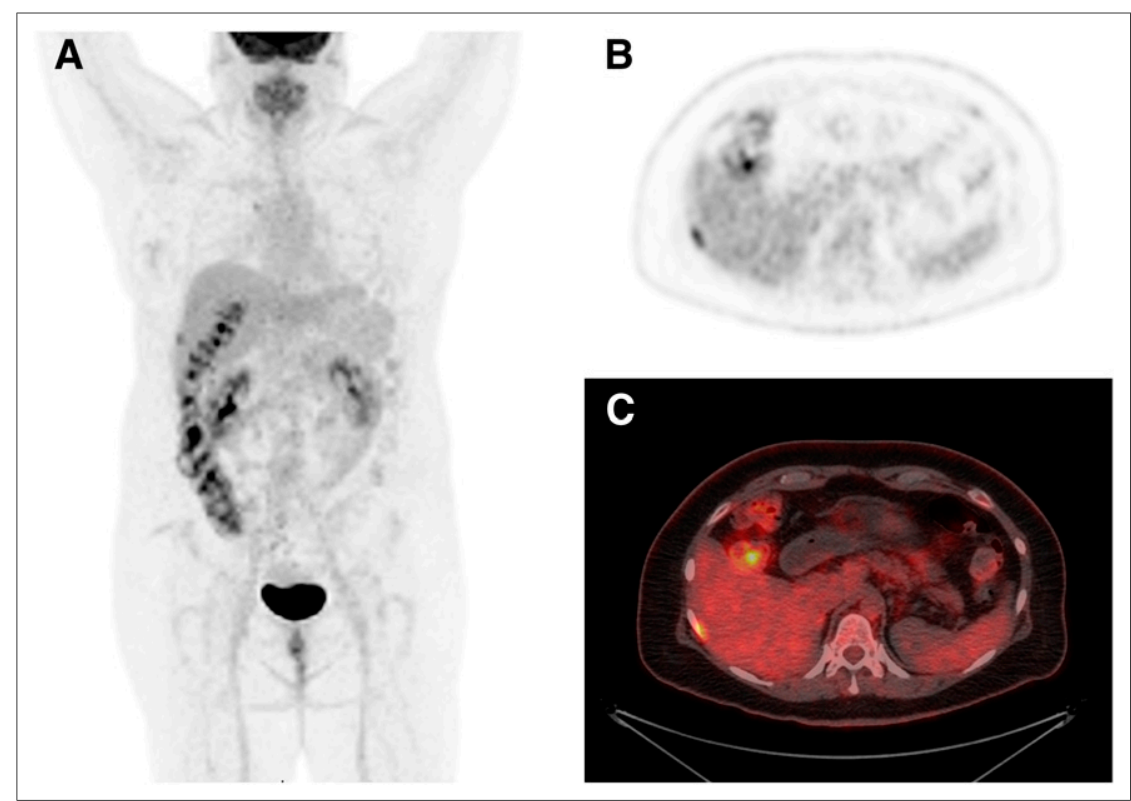

FIGURE 1. Anterior maximum-intensity projection (A) and axial ${ }^{18} \mathrm{~F}-\mathrm{FDG}$ PET (B) and $\mathrm{PET} / \mathrm{CT}(\mathrm{C})$ images show focal hypermetabolic activity on right side between liver and pleura. available in all labs even if not equipped with the latest generation of PET/ CT scanners.

\section{DISCLOSURE}

No potential conflict of interest relevant to this article was reported.

\section{REFERENCES}

1. Robertson IJ, Hand F, Kell MR. FDG-PET/CT in the staging of local/regional metastases in breast cancer. Breast. 2011;20:491-494.

2. Liu C, Pierce LA, Alessio AM, Kinahan PE. The impact of respiratory motion on tumor quantification and delineation in static PET/CT imaging. Phys Med Biol. 2009;54:7345-7362.

3. Quantitative nuclear medicine imaging: concepts, requirements and methods. International Atomic Energy Agency website. https://www.iaea.org/publications/10380/quantitative-nuclear-medicine-imaging-concepts-requirements-and-methods. Published 2014. Accessed January 5, 2021.

4. De Ponti E, Morzenti S, Crivellaro C, Elisei F, Crespi A, Guerra L. Motion management in PET/ CT: technological solutions. Curr Radiopharm. 2018; 11:79-85.

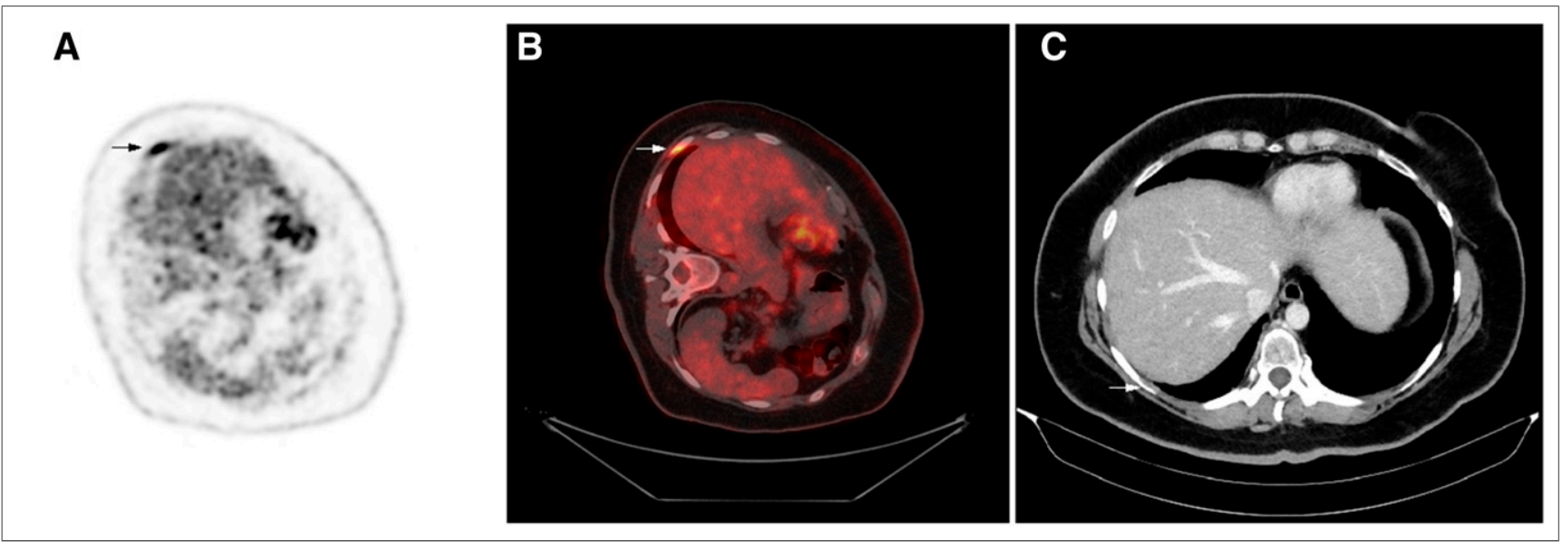

FIGURE 2. Axial maximum-intensity projection $(A)$ and axial PET/CT (B) image with patient positioned on right side show that lesion (arrows) was pleural; subsequently, high-dose CT (C) confirmed pleural location. 